\title{
CXCR5 overexpression in HL-60 cells enhances chemotaxis toward CXCL13 without anticipated interaction partners or enhanced MAPK signaling
}

\author{
Robert J. MacDonald ${ }^{1}$ Andrew Yen ${ }^{1}$ \\ ${ }^{1}$ Department of Biomedical Sciences, Cornell University College of Veterinary Medicine, \\ Veterinary Research Tower T4008A, Box 11, Ithaca, NY 14853, USA
}

\begin{abstract}
CXCR5 is a serpentine receptor implicated in cell migration in lymphocytes and differentiation in leukocytes. It causes MAPK pathway activation and has known membrane partners for signaling. CXCR5 mRNA is reportedly expressed in neutrophils following isolation, but its role in this cellular context is unknown. CXCR5 is also expressed in HL-60 cells, a human acute myeloid leukemia line, following treatment with all-trans retinoic acid, which induces differentiation toward a neutrophil-like state. CXCR5 is necessary for this process; differentiation was crippled in CXCR5 knockout cells and enhanced in cells ectopically expressing it. Since CXCR5 has various membrane protein partners, we investigated whether CXCR5-driven all-trans retinoic acid-induced differentiation depends on its association with such partners. Pursuing this, we generated HL-60 cells overexpressing the protein. We found that CXCR5 drove migration toward its ligand, CXCL13, and probed for interactions with several candidates using flow cytometry-based Förster resonance energy transfer. Surprisingly, we did not detect interactions with any candidates, including three reported in other cellular contexts. Additionally, we observed no significant changes in all-trans retinoic acid-induced differentiation; this may be due to the stoichiometry of CXCR5 and partner receptors or CXCL13. The anticipated membrane partnerings were surprisingly apparently unnecessary for downstream CXCR5 signaling and all-trans retinoic acidinduced differentiation.
\end{abstract}

\section{Keywords}

All-trans retinoic acid; Leukemia; CXCR5; Differentiation

\section{Introduction}

CXCR5 was originally discovered as Burkitt's lymphoma receptor 1 (BLR1), in a subtractive hybridization screen of Epstein-Barr virus-immortalized B cells subtracted from metastatic Burkitt's lymphoma cells to find determinants of the metastatic phenotype (Dobner et al. 1992). It was also later found by differential display to also be differentially

Andrew Yen, ay13@cornell.edu.

Conflict of interest The authors declare that they have no conflicts of interest. 
expressed in myeloid series cells as a function of differentiation (Battle et al. 2000). CXCR5 is a 7-pass putative heterotrimeric G-protein-coupled receptor capable of instigating mitogen-activated protein kinase (MAPK) pathway signaling (Dobner et al. 1992; Förster et al. 1996; Battle et al. 2000).

In lymphocytes, CXCR5 is essential for naïve B cell migration to follicles in lymph nodes and the spleen, where its ligand, CXCL13, is produced by stromal cells residing in these locations (Förster et al. 1996; Legler et al. 1998; Suto et al. 2009; Bénézech et al. 2015). In addition to B cells, CXCR5 is also highly expressed on T follicular helper cells (Breitfeld et al. 2000; Schaerli et al. 2000). One possible explanation for these expression patterns is that CXCL13 helps to divide the B cell and T cell zones in the lymph nodes; the T follicular helper cells may interact with follicular B cells to activate them and form germinal centers (Ansel et al. 2000; Kim et al. 2004; Moser 2015).

In myeloid series cells, CXCR5 mRNA expression has been detected in neutrophils following isolation and incubation for $3 \mathrm{~h}$ at $37^{\circ} \mathrm{C}$, though the consequences of expression of the protein was unclear (Patel et al. 2001). It is potentially noteworthy that expression of CXCL13 is upregulated following a number of infections, such as with Borrelia garinii, Chlamydia trachomatis, and Helicobacter pylori (Rupprecht et al. 2007; King et al. 2009; Nakashima et al. 2011). Additionally, CXCR5 is upregulated in HL-60 cells during all-trans retinoic acid (RA)-induced differentiation to neutrophil-like cells (Breitman et al. 1980; Battle et al. 2000. Hence, in myeloid cells, it has potential roles in response to infection and cell differentiation.

The HL-60 cell line, a human acute myeloid leukemia (AML) line, has been used as a model for all-trans retinoic acid (RA)-induced differentiation for several decades. The proteins and signaling pathways driving RA-induced differentiation, however, remain enigmatic. A previous report demonstrated that CXCR5 is necessary for the process, as bi-allelic knockout cripples differentiation (Wang and Yen 2008). Reports have also shown that ectopic expression of CXCR5 can enhance RA-induced differentiation, evidenced by increased activation of the MAPK signaling axis members c-Raf, MEK, and ERK; enhancement of $\mathrm{G}_{1} / \mathrm{G}_{0}$ cell cycle arrest; enhancement of the phenotypic marker of differentiation, CD11b; and enhancement of the functional marker of differentiation, inducible oxidative metabolism (Battle et al. 2000; Wang and Yen 2004, 2008).

Mechanistic details concerning signaling via CXCR5 remain unclear. Activation of CXCR5 by CXCL13 is characterized by chemotaxis toward CXCL13, a transient increase in intracellular calcium, and ERK signaling (Müller and Lipp 2001). The steps directly linking CXCR5 to the generation of a MAPK signal, however, are unknown. In the context of several prostate cancer cell lines, CXCR5 has been shown to co-immunoprecipitate with CXCR4 as well as $\mathrm{G}_{\mathrm{ai2} 2}, \mathrm{G}_{\mathrm{aq} / 11}, \mathrm{G}_{\mathrm{a} 13}, \mathrm{G}_{\beta 3}$, and $\mathrm{G}_{\gamma 9}$ (El-Haibi et al. 2013). CXCR5 has also been shown to homodimerize and to heterodimerize with EBI2 in HEK293T cells cotransfected to express the proteins (Barroso et al. 2012). The limited data thus suggest that CXCR5 might be following classical receptor activation paradigms regarding membrane and cytosolic partnering events. 
We sought to fill in gaps in understanding of CXCR5 signaling by searching for interaction partners following the classical premise that receptor dimerization can be seminal to activation and signaling. We generated HL-60 transfectants overexpressing CXCR5 $\left(\mathrm{CXCR}^{+}\right)$and found that the protein was active, evidenced by chemotaxis toward CXCL13. We then proceeded to probe for several candidate interaction partners found on the cell surface as well as two found in the cytosol. These interactions were probed using a previously established and validated flow cytometry-based Förster resonance energy transfer (FRET) method (MacDonald et al. 2017). Our membrane protein candidates were CD11b, CD38, CXCR4, CXCR5 itself, and EBI2. CD11b and CD38 are both cell surface markers that are upregulated during RA-induced differentiation (Hickstein et al. 1989; Kontani et al. 1993). CD38, while not necessary for RA-induced differentiation, enhances the process when overexpressed (Lamkin et al. 2006; MacDonald et al. 2017). CXCR4, CXCR5, and EBI2 were intended to be controls, as these interactions were previously reported in different cellular contexts (Barroso et al. 2012; El-Haibi et al. 2013). Our two cytosolic candidates, c$\mathrm{Cbl}$ and Lyn, have both been shown to play important roles in RA-induced differentiation (Shen and Yen 2008; Congleton et al. 2012). Surprisingly, we were unable to detect interactions between CXCR5 and any of our candidates, including the three previously reported interactions found in different cellular contexts (Barroso et al. 2012; El-Haibi et al. 2013). Unlike previous reports, the $\mathrm{CXCR}^{+}$cells did not exhibit enhanced RA-induced differentiation or associated signaling, suggesting a stoichiometric dependence between CXCR5 and CXCL13 or interaction partners. We did not detect CXCR5 receptor interactions with other membrane partners amongst our ensemble of putative candidates that might contribute to receptor dimerization-mediated signaling in HL-60 cells. Having eliminated dimerization with various candidates as a modality for CXCR5-driven signaling, additional studies to elucidate the important role of CXCR5 in RA-induced differentiation and its potential role in neutrophil function are needed.

\section{Materials and Methods}

\section{Cell culture}

HL-60 human myeloblastic leukemia cells, a generous gift of Dr. Robert Gallagher, were derived from the original patient isolates and maintained as previously described (MacDonald et al. 2017). The line was certified and tested for mycoplasma (Bio-Synthesis, Lewisville, TX) in August 2017. Raji cells were a kind gift of Dr. Kristi Richards and were maintained in RPMI 1640 supplemented with 10\% heat-inactivated FBS (GE Healthcare, Chicago, IL) and 1\% antibiotic/antimycotic (Thermo Fisher Scientific, Waltham, MA). HL-60 cells transfected to overexpress CXCR5 $\left(\mathrm{CXCR}^{+}\right)$were maintained as HL-60 cells following selection described in the "Generation of stable transfectants" section. RA was used at a final concentration of $1 \mu \mathrm{M}$ in keeping with established literature (Breitman et al. 1980).

\section{Antibodies and reagents}

Reagents, unless otherwise specified, were purchased from commercial suppliers in the highest purity available and used as supplied. PE-conjugated CD38, APC-conjugated CD11b, purified CD38, purified CD11b, CXCR4, and c-Raf antibodies were purchased from 
Becton Dickinson (Franklin Lakes, NJ). Alexa Fluor 488-conjugated CXCR5, purified CXCR5, and EBI2 antibodies were purchased from BioLegend (San Diego, CA). Lyn antibody was purchased from Abcam (Cambridge, UK). c-Raf pS621 antibody was purchased from Sigma (St. Louis, MO). c-Cbl antibody was purchased from Santa Cruz Biotechnology (Santa Cruz, CA). Total MEK1/2, phospho-MEK1/2 (S217/S221), total ERK1/2, phospho-ERK1/2 (T202/T404), and GAPDH antibodies were purchased from Cell Signaling Technologies (Danvers, MA).

\section{Generation of stable transfectants}

Human CXCR5 cDNA (NCBI reference sequence NM_001716.4), originally obtained from Genecopoeia (Rockville, MD) in the EX-A1294-M55 vector, was cloned into pEF1a-IRESNeo, a gift from Thomas Zwaka (plasmid no. 28019 from Addgene, Cambridge, MA). CXCR 5 was mobilized by PCR using PCR Supermix High Fidelity (Invitrogen, Carlsbad, CA) on a PTC-100 thermocycler (MJ Research, Waltham, MA). Primers to add N-terminal NheI and C-terminal Not restriction sites were purchased from Integrated DNA Technologies (Coralville, IA) (NheI: 5' TATGCCTAGCATGAACTACCCGCTAACGCTG-3', Not: $5^{\prime}$ TATAGCGGCCGCCTAGAACGTGGTGAGAGAGGT-3'). The product was purified using a PureLink PCR Purification Kit (Invitrogen) following the manufacturer's protocol. NheI and NotI were purchased from New England Biolabs (NEB) (Ipswich, MA) and used according to the manufacturer's protocol to digest the purified PCR product and pEF1aIRES-Neo. The cut insert and vector were then ligated using T4 DNA ligase (NEB), amplified in One Shot Top10 competent cells (Thermo Fisher Scientific, Waltham, MA), and purified using QIAprep Miniprep kits (Qiagen, Hilden, Germany), all according to manufacturer's instructions. Purified plasmid was sequenced to confirm appropriate $C X C R 5$ insertion.

To generate $\mathrm{CXCR}^{+}$, stable HL-60 transfectants overexpressing CXCR5, transfection was performed as previously described (Congleton et al. 2012). CXCR5 ${ }^{+}$cells were selected for 2 weeks in RPMI 1640 supplemented with $10 \%$ heat-inactivated fetal bovine serum and 1 $\mathrm{mg} / \mathrm{mL}$ G418. CXCR5 ${ }^{+}$cells were then purified via FACS using a Becton Dickinson FACS Aria III SORP (Becton Dickinson, Franklin Lakes, NJ) following immunostaining with an Alexa Fluor 488-conjugated anti-CXCR5 antibody (BioLegend, San Diego, CA) as previously described for CD11b (MacDonald et al. 2017). Gating for sorting was set to include cells with fluorescence levels equal to or greater than the top $1 \%$ of stained wildtype HL-60 cells in the appropriate channel. Gating was set to exclude doublets and autofluorescent cells by excluding cells that did not have roughly 1:1 values along a forward scatter area vs forward scatter height plot and by excluding cells with fluorescence in channels other than Alexa Fluor 488 emission. To validate stable transfection, CXCR5 levels in $\mathrm{CXCR}^{+}$, Raji (positive control), HL-60 (negative control), and 48-h RA-treated HL-60 cells were analyzed by flow cytometry. As previously reported, mock transfection had no effect on the proliferation or differentiation of these cells and was not repeated here (Platko et al. 1998). 


\section{Cell migration assay}

To determine propensity of different cells to migrate toward CXCL13, the ligand for CXCR5, we used 6.5-mm Transwell inserts with 5.0- $\mu \mathrm{M}$ pore sizes in 24-well plates from Corning (Corning, NY) using a previously described migration assay (Lee et al. 2006). Briefly, $600 \mu \mathrm{L}$ of complete culture media with $0,0.1,0.5$, or $2.5 \mu \mathrm{g} / \mathrm{ml}$ human recombinant CXCL13, purchased from BioLegend (San Diego, CA), was added to the bottom of each well. After $48 \mathrm{~h}$ of culture with (for HL-60) or without (for HL-60 and CXCR5 ${ }^{+}$) RA treatment, $1.5 \times 105$ cells were added to the inserts and the plate was incubated at $37^{\circ} \mathrm{C}$ with $5 \% \mathrm{CO}_{2}$ for $6 \mathrm{~h}$. The inserts were then removed and wells were imaged using a BioRad ZOE fluorescent cell imager (Hercules, CA). Cells that had traveled through the membrane to the well were resuspended by pipetting, transferred to microcentrifuge tubes, and pelleted. Media was removed and cells were resuspended in $100 \mathrm{uL}$ phosphate-buffered saline and counted using a hemocytometer. To determine percentage of cell migration for each sample, the total number of cells that had migrated to the wells was divided by the number of input cells and this proportion was multiplied by 100 . We assumed negligible cell proliferation, as well as negligible differences in cell proliferation across samples, during the 6-h treatment period. Three biological replicates were performed.

\section{Förster resonance energy transfer experiments}

We probed for CXCR5 interaction partners using a flow cytometry-based FRET method previously described and used to successfully detect CD38 homodimerization in HL-60 cells following treatment with RA and/or a synthetic CD38 linker (Förster 1948; MacDonald et al. 2017). Antibody conjugation for Alexa Fluor 546 conjugates (c-Cbl, CD11b, and Lyn) was performed as previously described (MacDonald et al. 2017). Alexa Fluor 555 conjugates (CD38, CXCR4, CXCR5, and EBI2) were created using an Alexa Fluor 555 antibody labeling kit from Thermo Fisher Scientific following the manufacturer's protocol. We chose to use these fluors for the acceptor as they are more sensitive than the Alexa Fluor 488/Alexa Fluor 594 pair according to the manufacturer. Although our experimental setup was largely performed according to the previously described method (MacDonald et al. 2017), we adjusted our filter and mirror sets to accommodate Alexa Fluor 546 and Alexa Fluor 555, collecting through a 550-nm-longpass dichroic mirror and a 585/42-nm filter. When running samples probing for interactions between CXCR5 and CD11b, CD38, CXCR4, CXCR5, and EBI2, live samples were run. When running samples probing for interactions between CXCR5 and c-Cbl and Lyn, two cytosolic proteins, cells were fixed prior to staining, as previously described (Bunaciu et al. 2015). Three biological replicates were performed for each FRET pair.

\section{Phenotyping}

Immunostaining for $\mathrm{CD} 11 \mathrm{~b}$ and $\mathrm{CD} 38$ was performed as previously described and analyzed using a Becton Dickinson LSR II flow cytometer (MacDonald et al. 2017). Propidium iodide (PI) cell cycle analysis was performed as previously described (MacDonald et al. 2017). Three biological replicates were performed. 


\section{Western blot analysis}

Western blots were performed as previously described (MacDonald et al. 2017) and representative blots of three biological replicates are shown.

\section{Statistics}

Data were analyzed using GraphPad Prism version 7.02. One-way or two-way analysis of variance (ANOVA) was used to determine significance as appropriate. Error bars indicate mean \pm standard deviation.

\section{Results}

\section{Generation of stable transfectants overexpressing CXCR5}

To probe for CXCR5 binding partners, we generated stable HL-60 transfectants overexpressing CXCR5 $\left(\mathrm{CXCR}^{+}\right)$. CXCR5 ${ }^{+}$and HL-60 cells were treated with RA for $48 \mathrm{~h}$ as indicated in Fig. 1, a time when indications of overt differentiation evidenced by CD11b expression and $\mathrm{G}_{1} / \mathrm{G}_{0}$ arrest first become apparent (Yen et al. 1985; Hickstein et al. 1989). HL-60 cells do not express CXCR5, and as shown in Fig. 1, there is minimal background fluorescence; expression is mildly but significantly induced by RA treatment. The percentage of positive cells is much lower than that of the positive control, Raji cells, which are $100 \%$ positive (Kanbe et al. 1999; Battle et al. 2000). CXCR5 ${ }^{+}$cells are $100 \%$ positive like Raji. CXCR5 ${ }^{+}$cells have significantly greater CXCR5 per cell, measured by mean fluorescence per cell, than the Raji cells. The CXCR5 stable transfection thus achieved a high level of expression.

\section{CXCR5 ${ }^{+}$cells migrate toward CXCL13}

To determine that the CXCR5 expressed in the $\mathrm{CXCR}^{+}$cell line was functional, we performed a cell migration assay testing for migration toward CXCL13, the ligand of CXCR5 (Ebert et al. 2005). We compared migration of HL-60 cells, which do not express CXCR5, to that of RA-treated HL-60 cells (to induce CXCR5 expression) and CXCR5 ${ }^{+}$ cells. Following a previously reported experimental protocol for CXCL13-mediated migration, we measured the percentage of cells that migrated from Transwell inserts to wells below containing varying concentrations of CXCL13 in complete growth media (Lee et al. 2006). As shown in Fig. 2, we used concentrations between 0 and $2.5 \mu \mathrm{g} / \mathrm{mL}$ and did not observe chemotaxis in untreated HL-60 cells, which do not express CXCR5, over the 6-h incubation period. RA-treated HL-60 cells, which express CXCR5, migrated at low levels under all conditions, with increased migration at $0.5 \mu \mathrm{g} / \mathrm{mL} \mathrm{CXCL13.} \mathrm{CXCR5}{ }^{+}$cells did not migrate without ligand, migrated minimally at $0.1 \mu \mathrm{g} / \mathrm{mL} \mathrm{CXCL13,} \mathrm{and} \mathrm{had} \mathrm{the} \mathrm{highest}$ degree of migration with $0.5 \mu \mathrm{g} / \mathrm{mL}$ CXCL13. For both RA-treated HL-60 cells and $\mathrm{CXCR}^{+}$cells, migration decreased with $2.5 \mu \mathrm{g} / \mathrm{mL}$ CXCL13 compared to $0.5 \mu \mathrm{g} / \mathrm{mL}$ CXCL13. The ectopic CXCR5 ergo appears to be functional.

\section{No detectable FRET signal between CXCR5 and membrane protein candidates}

We used a previously established and validated flow cytometry-based FRET assay to test whether CXCR5 interacts with CD11b, CD38, CXCR4, CXCR5, and EBI2. We chose this 
set of candidates based on their upregulation in RA-induced differentiation and their previously reported interactions in other cellular contexts (Hickstein et al. 1989; Kontani et al. 1993; Barroso et al. 2012; El-Haibi et al. 2013). As shown in Fig. 3, HL-60 and CXCR5 ${ }^{+}$ cells were treated for $24 \mathrm{~h}$ with RA as indicated and FRET measurements were performed using the Alexa Fluor 488/Alexa Fluor 546 and Alexa Fluor 488/Alexa Fluor 555 pairs. We observed no enhancement of signal in the FRET channels above background, unstained levels; hence, there was no FRET-measured dimerization of CXCR5 with any of the anticipated membrane partners.

\section{No detectable FRET signal between CXCR5 and cytosolic protein candidates, $\mathrm{c}-\mathrm{Cb}$ and Lyn}

We used flow cytometry-based FRET to test whether CXCR5 interacts with c-Cbl and Lyn, two cytosolic proteins important for RA-induced differentiation. CXCR5 has been found to cause MAPK signaling and c-Cbl and Lyn are known regulators of MAPK signaling pathways (Battle et al. 2000; Wei et al. 2000; Swaminathan and Tsygankov 2006; Congleton et al. 2012). Both proteins are present in a signalsome driven by MAPK signaling dependent on CXCR5 (Shen and Yen 2008; Congleton et al. 2012). HL-60 and CXCR5 ${ }^{+}$cells with or without 24-h RA treatment were analyzed. As shown in Fig. 4, we observed no enhancement of signal in the FRET channels above background, unstained levels. Since the typical detectable FRET distance is approximately $10 \mathrm{nM}$, this indicates there was no immediate association of CXCR5 and these candidates (Shrestha et al. 2015).

\section{Differentiation-associated signaling protein expression is not affected by CXCR5 overexpression}

We sought to test whether CXCR5 overexpression might affect members of an ensemble of MAPK proteins-RA-induced differentiation is driven by a signalsome that incorporates a c-Raf/MEK/ERK axis that undergoes sustained activation and drives differentiation (Yen et al. 1998). We treated HL-60 and CXCR5 ${ }^{+}$cells for $48 \mathrm{~h}$ with or without $1 \mu \mathrm{M}$ RA, collected total cell lysate, and performed western blots probing for c-Raf, MEK, ERK, and their activated phosphoproteins. We did not observe differences in expression or phosphorylation of the proteins assessed between HL-60 and CXCR $5^{+}$cells; representative blots are shown in Fig. 5. Levels of total MEK and total ERK remained constant following RA treatment, whereas levels of total c-Raf, c-Raf phosphorylated at S621, phosphorylated MEK, and phosphorylated ERK were enhanced. GAPDH was used as a loading control.

\section{Overexpression of CXCR5 does not alter RA-induced differentiation}

We characterized the effects of high levels of ectopic CXCR5 on RA-induced differentiation. We compared RA-induced differentiation in HL-60 cells to that of $\mathrm{CXCR}^{+}$cells over a 72$\mathrm{h}$ treatment period, using several myeloid lineage markers as indicators of maturation: CD38 and $\mathrm{CD} 11 \mathrm{~b}$ cell surface expression, $\mathrm{G}^{1} / \mathrm{G}_{0}$ cell cycle arrest, and cell density. We used several different concentrations of RA, from 0.1 to the standard $1 \mu \mathrm{M}$, in order to capture effects of CXCR5 overexpression that may only be apparent when using weaker RA stimulation.

As shown in Fig. 6, while some of the markers, such as CD11b, exhibited a dose-response relationship with varying concentrations of RA, there were no significant differences 
between HL-60 and $\mathrm{CXCR}^{+}$cells. CD38 levels after $8 \mathrm{~h}$ of treatment were higher in the $\mathrm{CXCR}^{+}$cells, but these differences are not statistically significant. Interestingly, there was a slight, albeit not statistically significant, enhancement of cell density/growth in RA-treated cells associated with ectopic CXCR5 expression. This is consistent with the other data indicating functionality of the ectopic CXCR5, but the molecular mechanism here is enigmatic.

\section{Discussion}

While CXCR5 is capable of generating a MAPK signal, the mechanistic details are not known. Such an understanding is of import to the biology of cell proliferation and differentiation. Previous reports have implicated the protein in RA-induced differentiation of HL-60 cells through its ability to activate MAPK signaling (Battle et al. 2000; Wang and Yen 2008). A durable MAPK signaling is known to be necessary to drive cell cycle arrest and differentiation (Traverse et al. 1992; Yen et al. 1994, 1998; Yen and Varvayanis 2000). Knocking out CXCR5 cripples differentiation, and ectopic expression can enhance it (Battle et al. 2000; Wang and Yen 2004, 2008). Here, we sought to explore binding partners of CXCR5 that may help to explain its role in RA-induced differentiation. Given that dimerization is one of the classical means of starting receptor signaling, we explored the conjecture that RA-induced proteins might collaborate with CXCR5 by dimerization to generate the sustained MAPK signaling involved in RA-induced differentiation.

We first created stable HL-60 transfectants overexpressing CXCR5, CXCR5 ${ }^{+}$cells. As shown in Fig. 1, CXCR5 ${ }^{+}$cells express CXCR5 at levels well above that of RA-treated HL-60 cells as well as of a positive control, the Raji cell line. We then established that the overexpressed CXCR5 was functional by showing it enhanced migration toward CXCL13a known attribute of the receptor. $\mathrm{CXCR} 5^{+}$cells demonstrated strong chemotaxis toward CXCL13, especially at the $0.5 \mu \mathrm{g} / \mathrm{mL}$ concentration, as shown in Fig. 2. HL-60 cells, which do not express CXCR5, did not migrate toward the ligand. When treated with RA to induce modest expression of CXCR5, HL-60 cells exhibited a milder degree of migration with a dose-response curve similar to that of the $\mathrm{CXCR}^{+}$cells. There was a background level of migration in RA-treated HL-60 cells; however, this may be due to expression of a number of other cell surface receptors that could drive migration toward other factors in the media. Interestingly, the propensity of $\mathrm{CXCR}^{+}$and RA-treated HL-60 cells to migrate toward CXCL13 diminished at the highest dose of CXCL13 tested, suggesting that activity in response to CXCL13 may follow a bell-shaped curve. The results suggest that the CXCR5 receptor may function - as do other receptors dependent, for example, on partners and relative stoichiometry therewith—in a dose-dependent manner.

We next probed a set of candidate interaction partners in an attempt to determine if a classical receptor dimerization process was connecting CXCR5 to downstream MAPK signaling. We performed several flow cytometry-based FRET experiments, shown in Figs. 3 and 4, using a previously established experimental protocol for detecting protein dimerization (Förster 1948). This method, based on directly conjugating Alexa Fluor dyes to primary antibodies against targets of interest, detected CD38 dimerization as described in a previous report (MacDonald et al. 2017). However, we did not detect interactions between 
CXCR5 and any of our candidates, a combination of surface markers and downstream proteins implicated in RA-induced differentiation or shown to interact with CXCR5 in other cellular contexts (Hickstein et al. 1989; Kontani et al. 1993; Lamkin et al. 2006; Shen and Yen 2008; Barroso et al. 2012; Congleton et al. 2012; El-Haibi et al. 2013). The candidate cell surface proteins were either implicated in other contexts or RA-induced moieties that might rationalize prolonged signal duration. The putative downstream signaling candidates tested, c-Cbl and Lyn, are regulators of the MAPK signaling pathway, the central cRaf/MEK/ERK axes of which undergoes prolonged activation by RA to drive differentiation (Traverse et al. 1992; Yen et al. 1994, 1998; Wei et al. 2000; Yen and Varvayanis 2000; Swaminathan and Tsygankov 2006). The absence of FRET argues against a direct association, but it does not preclude interactions of partners indirectly connected at a distance exceeding the Förster radius.

Our $\mathrm{CXCR}^{+}$cell line did not behave as previous ectopic CXCR5 expressing lines had; we saw no phenotypic enhancement of RA-induced differentiation or associated signaling, as shown in Figs. 5 and 6 (Battle et al. 2000; Wang and Yen 2004, 2008). Several factors may explain the differences in behavior of the presently generated cell line compared to previously described CXCR5-expressing lines. Although we used a vector with a design similar to those previously used, with an EF1a promoter and an internal ribosomal entry site linked to a neomycin resistance gene, the two were not identical and we do not know how the expression levels of CXCR5 in the previous papers compare to those in the current report (Battle et al. 2000; Wang and Yen 2004, 2008). Based on the comparisons to the Raji cell line and RA-treated HL-60 cells, the levels of CXCR5 expressed in the CXCR5 ${ }^{+}$line are very high. It is possible that the dose-response for CXCR5 receptor signaling activity and consequential cellular effects may follow a bell-shaped curve, such that low and high levels of the receptor in the presence of a given amount of ligand have little effect but moderate levels may enhance its activity and, in turn, RA-induced differentiation. This shape of doseresponse curve, while it is not indicative, can be a feature of ligand-induced receptor dimerization (Atanasova and Whitty 2012). An optimum dose for eliciting a given effect may be a telltale of complex multi-molecular signaling complexes (Onfroy et al. 2017). Differential stoichiometric effects of CXCR5 with different signaling partners could contribute to potentially complex dose-dependent effects. Additionally, it is possible that serum levels of the CXCR5 ligand, CXCL13, were not identical in the present and previous reports (Battle et al. 2000; Wang and Yen 2004, 2008). CXCL13 levels in fetal bovine serum are not routinely assessed by manufacturers, and bovine and human CXCL13 share over $50 \%$ sequence identity, so it is plausible that bovine CXCL13 may be able to act as a ligand for human CXCR5 (Bateman et al. 2017).

\section{Conclusions}

Here, we report that FRET revealed no interaction between CXCR5 and a small ensemble of candidates that may have helped explain its role in RA-induced differentiation in HL-60 cells. This includes the surface receptors CD11b and CD38, the cytosolic Src family kinase Lyn, the scaffolding protein and E3 ubiquitin ligase c-Cbl, and several partners previously reported in different cellular contexts: CXCR4, EBI2, and CXCR5 itself (Barroso et al. 2012; El-Haibi et al. 2013). Our results suggest that CXCR5 may not directly interact with 
these proteins in the HL-60 context. Given its role in RA-induced differentiation and that its ligand, CXCL13, is overexpressed in the context of a variety of infections, including those of Helicobacter pylori, Treponema pallidum, and Borrelia spp., greater understanding of CXCR5 signaling is of significance in that it may ultimately yield actionable targets for therapeutic use (Rupprecht et al. 2007; Marra et al. 2010; Nakashima et al. 2011; Schmidt et al. 2011; Zeng et al. 2016). Further studies that extend the present report to elucidate the mechanism by which CXCR5 is able to generate a downstream MAPK signal are thus called for.

\section{Acknowledgments}

Funding information This work was supported by grant R01 CA152870 from the National Institute of Health (Yen). The content is solely the responsibility of the authors and does not necessarily represent the official views of the National Institutes of Health.

\section{References}

Ansel KM, Ngo VN, Hyman PL, Luther SA, Förster R, Sedgwlck JD, Browning JL, Upp M, Cyster JG (2000) A chemokine-driven positive feedback loop organizes lymphoid follicles. Nature 406:309314. 10.1038/35018581 [PubMed: 10917533]

Atanasova M, Whitty A (2012) Understanding cytokine and growth factor receptor activation mechanisms. Crit Rev Biochem Mol Biol 47:502-530. 10.3109/10409238.2012.729561 [PubMed: 23046381]

Barroso R, Martínez Muñoz L, Barrondo S, Vega B, Holgado BL, Lucas P, Baíllo A, Sallés J, Rodríguez-Frade JM, Mellado M (2012) EBI2 regulates CXCL13-mediated responses by heterodimerization with CXCR5. FASEB J 26:4841-4854. 10.1096/fj.12-208876 [PubMed: 22913878]

Bateman A, Martin MJ, O’Donovan C, Magrane M, Alpi E, Antunes R, Bely B, et al. (2017) UniProt: the universal protein knowledgebase. Nucleic Acids Res 45:D158-D169. 10.1093/nar/gkw1099 [PubMed: 27899622]

Battle TE, Levine RA, Yen A (2000) Retinoic acid-induced blr1 expression promotes ERK2 activation and cell differentiation in HL-60 cells. Exp Cell Res 254:287-298. 10.1006/excr.1999.4766 [PubMed: 10640427]

Bénézech C, Luu NT, Walker JA, Kruglov AA, Loo Y, Nakamura K, Zhang Y, Nayar S, Jones LH, Flores-Langarica A, McIntosh A, Marshall J, Barone F, Besra G, Miles K, Allen JE, Gray M, Kollias G, Cunningham AF, Withers DR, Toellner KM, Jones ND, Veldhoen M, Nedospasov SA, McKenzie ANJ, Caamaño JH (2015) Inflammation-induced formation of fat-associated lymphoid clusters. Nat Immunol 16:819-828. 10.1038/ni.3215 [PubMed: 26147686]

Breitfeld D, Ohl L, Kremmer E, Ellwart J, Sallusto F, Lipp M, Förster R (2000) Follicular B helper T cells express CXC chemokine receptor 5, localize to B cell follicles, and support immunoglobulin production. J Exp Med 192:1545-1552. 10.1084/jem.192.11.1545 [PubMed: 11104797]

Breitman TR, Selonick SE, Collins SJ (1980) Induction of differentiation of the human promyelocytic leukemia cell line (HL-60) by retinoic acid. Proc Natl Acad Sci U S A 77:2936-2940. 10.1073/ pnas.77.5.2936 [PubMed: 6930676]

Bunaciu RP, Jensen HA, MacDonald RJ, LaTocha DH, Varner JD, Yen A (2015) 6-Formylindolo(3,2b)carbazole (FICZ) modulates the signalsome responsible for RA-induced differentiation of HL-60 myeloblastic leukemia cells. PLoS One 10:e0135668 10.1371/journal.pone.0135668 [PubMed: 26287494]

Congleton J, MacDonald R, Yen A (2012) Src inhibitors, PP2 and dasatinib, increase retinoic acidinduced association of Lyn and c-Raf (S259) and enhance MAPK-dependent differentiation of myeloid leukemia cells. Leukemia 26:1180-1188. 10.1038/leu.2011.390 [PubMed: 22182854] 
Dobner T, Wolf I, Emrich T, Lipp M (1992) Differentiation-specific expression of a novel G proteincoupled receptor from Burkitt's lymphoma. Eur J Immunol 22:2795-2799. 10.1002/eji. 1830221107 [PubMed: 1425907]

Ebert LM, Schaerli P, Moser B (2005) Chemokine-mediated control of T cell traffic in lymphoid and peripheral tissues. Mol Immunol 42:799-809. 10.1016/j.molimm.2004.06.040 [PubMed: 15829268]

El-Haibi CP, Sharma P, Singh R, Gupta P, Taub DD, Singh S, Lillard JW (2013) Differential G protein subunit expression by prostate cancer cells and their interaction with CXCR5. Mol Cancer 12:64 10.1186/1476-4598-12-64 [PubMed: 23773523]

Förster R, Mattis AE, Kremmer E, Wolf E, Brem G, Lipp M (1996) A putative chemokine receptor, BLR1, directs B cell migration to defined lymphoid organs and specific anatomic compartments of the spleen. Cell 87:1037-1047. 10.1016/S0092-8674(00)81798-5 [PubMed: 8978608]

Förster T (1948) Intermolecular energy transference and fluorescence. Ann Phys 2:55-75

Hickstein DD, Back AL, Collins SJ (1989) Regulation of expression of the CD11b and CD18 subunits of the neutrophil adherence receptor during human myeloid differentiation. J Biol Chem 264:21812-21817 [PubMed: 2600090]

Kanbe K, Shimizu N, Soda Y, Takagishi K, Hoshino H (1999) A CXC chemokine receptor, CXCR5/ BLR1, is a novel and specific coreceptor for human immunodeficiency virus type 2. Virology 265:264-273. 10.1006/viro.1999.0036 [PubMed: 10600598]

Kim CH, Lim HW, Kim JR, Rott L, Hillsamer P, Butcher EC (2004) Unique gene expression program of human germinal center T helper cells. Blood 104:1952-1960. 10.1182/blood-2004-03-1206 [PubMed: 15213097]

King M, Poya H, Rao J, Natarajan S, Butch AW, Aziz N, Kok S, Chang MH, Lyons JM, Ault K, Kelly KA (2009) CXCL13 expression in chlamydia trachomatis infection of the female reproductive tract. Drugs Today (Barc) 45(Suppl B):125-134 [PubMed: 20011704]

Kontani K, Nishina H, Ohoka Y, Takahashi K, Katada T (1993) NAD glycohydrolase specifically induced by retinoic acid in human leukemic HL-60 cells. Identification of the NAD glycohydrolase as leukocyte cell surface antigen CD38. J Biol Chem 268:16895-16898 [PubMed: 8394323]

Lamkin TJ, Chin V, Varvayanis S, Smith JL, Sramkoski RM, Jacobberger JW, Yen A (2006) Retinoic acid-induced CD38 expression in HL-60 myeloblastic leukemia cells regulates cell differentiation or viability depending on expression levels. J Cell Biochem 97:1328-1338. 10.1002/jcb.20745 [PubMed: 16329108]

Lee BP-L, Chen W, Shi H, Der SD, Förster R, Zhang L (2006) CXCR5/CXCL13 interaction is important for double-negative regulatory $\mathrm{T}$ cell homing to cardiac allografts. J Immunol 176:5276-1583 [PubMed: 16621993]

Legler DF, Loetscher M, Roos RS, Clark-Lewis I, Baggiolini M, Moser B (1998) B cell-attracting chemokine 1, a human CXC chemokine expressed in lymphoid tissues, selectively attracts B lymphocytes via BLR1/CXCR5. J Exp Med 187:655-660. 10.1084/jem.187.4.655 [PubMed: 9463416]

MacDonald RJ, Shrimp JH, Jiang H, Zhang L, Lin H, Yen A (2017) Probing the requirement for CD38 in retinoic acid-induced HL-60 cell differentiation with a small molecule dimerizer and genetic knockout. Sci Rep 7:17406 10.1038/s41598-017-17720-4 [PubMed: 29234114]

Marra CM, Tantalo LC, Sahi SK, Maxwell CL, Lukehart SA (2010) CXCL13 as a cerebrospinal fluid marker for neurosyphilis in HIV-infected patients with syphilis. Sex Transm Dis 37:283-287. 10.1097/OLQ.0b013e3181d877a1 [PubMed: 20393380]

Moser B (2015) CXCR5, the defining marker for follicular B helper $\mathrm{T}\left(\mathrm{T}_{\mathrm{FH}}\right)$ cells. Front Immunol 6:296 10.3389/fimmu.2015.00296 [PubMed: 26106395]

Müller G, Lipp M (2001) Signal transduction by the chemokine receptor CXCR5: structural requirements for $\mathrm{G}$ protein activation analyzed by chimeric CXCR1/CXCR5 molecules. Biol Chem 382:1387-1397. 10.1515/BC.2001.171 [PubMed: 11688722]

Nakashima Y, Isomoto H, Matsushima K, Yoshida A, Nakayama T, Nakayama M, Hisatsune J, Ichikawa T, Takeshima F, Hayashi T, Nakao K, Hirayama T, Kohno S (2011) Enhanced expression 
of CXCL13 in human helicobacter pylori-associated gastritis. Dig Dis Sci 56:2887-2894. 10.1007/ s10620-011-1717-8 [PubMed: 21647655]

Onfroy L, Galandrin S, Pontier SM, Seguelas M-H, N'Guyen D, Sénard J-M, Galés C (2017) G protein stoichiometry dictates biased agonism through distinct receptor-G protein partitioning. Sci Rep 7:7885 10.1038/s41598-017-07392-5 [PubMed: 28801617]

Patel L, Charlton SJ, Chambers JK, Macphee CH (2001) Expression and functional analysis of chemokine receptors in human peripheral blood leukocyte populations. Cytokine 14:27-36. 10.1006/cyto.2000.0851 [PubMed: 11298490]

Platko JD, Forbes ME, Varvayanis S, Williams MN, Brooks SC, Cherington V, Yen A (1998) Polyoma middle T antigen in HL-60 cells accelerates hematopoietic myeloid and monocytic cell differentiation. Exp Cell Res 238:42-50. 10.1006/excr.1997.3782 [PubMed: 9457055]

Rupprecht TA, Kirschning CJ, Popp B, Kastenbauer S, Fingerle V, Pfister HW, Koedel U (2007) Borrelia garinii induces CXCL13 production in human monocytes through toll-like receptor 2. Infect Immun 75:4351-4356. 10.1128/IAI.01642-06 [PubMed: 17562761]

Schaerli P, Willimann K, Lang AB, Lipp M, Loetscher P, Moser B (2000) CXC chemokine receptor 5 expression defines follicular homing T cells with B cell helper function. J Exp Med 192:15531562. 10.1084/jem.192.11.1553 [PubMed: 11104798]

Schmidt C, Plate A, Angele B, Pfister H-W, Wick M, Koedel U, Rupprecht TA (2011) A prospective study on the role of CXCL13 in Lyme neuroborreliosis. Neurology 76:1051-1058. 10.1212/WNL. 0b013e318211c39a [PubMed: 21422457]

Shen M, Yen A (2008) c-Cbl interacts with CD38 and promotes retinoic acid-induced differentiation and G0 arrest of human myeloblastic leukemia cells. Cancer Res 68:8761-8769. 10.1158/0008-5472.CAN-08-1058 [PubMed: 18974118]

Shrestha D, Jenei A, Nagy P, Vereb G, Szöllősi J (2015) Understanding FRET as a research tool for cellular studies. Int J Mol Sci 16:6718-6756. 10.3390/ijms16046718 [PubMed: 25815593]

Suto H, Katakai T, Sugai M, Kinashi T, Shimizu A (2009) CXCL13 production by an established lymph node stromal cell line via lymphotoxin-beta receptor engagement involves the cooperation of multiple signaling pathways. Int Immunol 21:467-476. 10.1093/intimm/dxp014 [PubMed: 19251935]

Swaminathan G, Tsygankov AY (2006) The Cbl family proteins: ring leaders in regulation of cell signaling. J Cell Physiol 209:21-43. 10.1002/jcp.20694 [PubMed: 16741904]

Traverse S, Gomez N, Paterson H, Marshall C, Cohen P (1992) Sustained activation of the mitogenactivated protein (MAP) kinase cascade may be required for differentiation of PC12 cells. Comparison of the effects of nerve growth factor and epidermal growth factor. Biochem J 288(Pt 2):351-355. 10.1042/BJ2880351 [PubMed: 1334404]

Wang J, Yen A (2004) A novel retinoic acid-responsive element regulates retinoic acid-induced BLR1 expression. Mol Cell Biol 24:2423-2443. 10.1128/MCB.24.6.2423-2443.2004 [PubMed: 14993281]

Wang J, Yen A (2008) A MAPK-positive feedback mechanism for BLR1 signaling propels retinoic acid-triggered differentiation and cell cycle arrest. J Biol Chem 283:4375-4386. 10.1074/ jbc.M708471200 [PubMed: 18006504]

Wei S, Liu JH, Epling-Burnette PK, Jiang K, Zhong B, Elkabani ME, Pearson ME, Djeu JY (2000) IL-2 induces the association of IL-2Rbeta, lyn, and MAP kinase ERK-1 in human neutrophils. Immunobiology 202:363-382. 10.1016/S0171-2985(00)80040-6 [PubMed: 11131153]

Yen A, Varvayanis S (2000) Retinoic acid increases amount of phosphorylated RAF; ectopic expression of cFMS reveals that retinoic acid-induced differentiation is more strongly dependent on ERK2 signaling than induced GO arrest is. In Vitro Cell Dev Biol Anim 36:249-255. 10.1290/1071-2690(2000)036<0249:RAIAOP>2.3.CO;2 [PubMed: 10852350]

Yen A, Reece SL, Albright KL (1985) Control of cell differentiation during proliferation - II. Myeloid differentiation and cell cycle arrest of HL-60 promyelocytes preceded by nuclear structural changes. Leuk Res 9:51-71. 10.1016/0145-2126(85)90021-9 [PubMed: 3857406]

Yen A, Williams M, Platko JD, Der C, Hisaka M (1994) Expression of activated RAF accelerates cell differentiation and RB protein down-regulation but not hypophosphorylation. Eur J Cell Biol 65:103-113 [PubMed: 7889981] 
Yen A, Roberson MS, Varvayanis S, Lee AT (1998) Retinoic acid induced mitogen-activated protein (MAP)/extracellular signal-regulated kinase (ERK) kinase-dependent MAP kinase activation needed to elicit HL-60 cell differentiation and growth arrest. Cancer Res 58:3163-3172 [PubMed: 9679985]

Zeng YL, Lin YQ, Zhang NN, Zou CN, Zhang HL, Peng F, Liu ZJ, Zheng WH, Yan JH, Liu LL (2016) CXCL13 chemokine as a promising biomarker to diagnose neurosyphilis in HIV-negative patients. Springerplus 5:743 10.1186/s40064-016-2462-4 [PubMed: 27376011] 

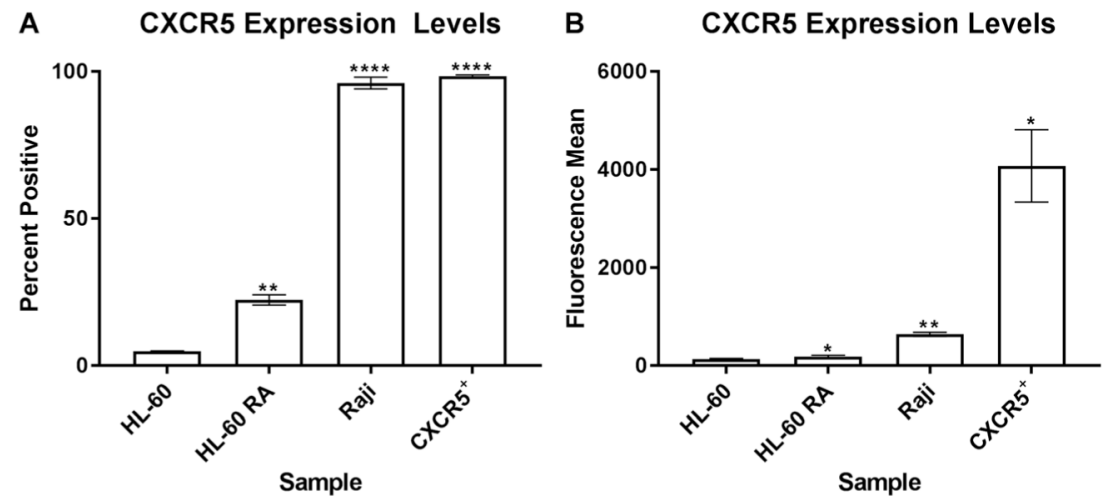

Figure 1.

$\mathrm{CXCR}^{+}$cells have high CXCR5 expression levels. $(A)$ CXCR5 expression levels in HL-60, RA-treated HL-60, Raji, and CXCR5 ${ }^{+}$cells were measured by flow cytometry after a 48-h treatment period. Gating was set to exclude $95 \%$ of stained control cells (HL-60). $n=3$. **, $p<0.01$; ****, $p<0.0001$. (B) CXCR5 expression levels shown in terms of mean fluorescence levels. ${ }^{*}, p<0.05 ; * *, p<0.01$. Error bars indicate standard deviation (SD). 


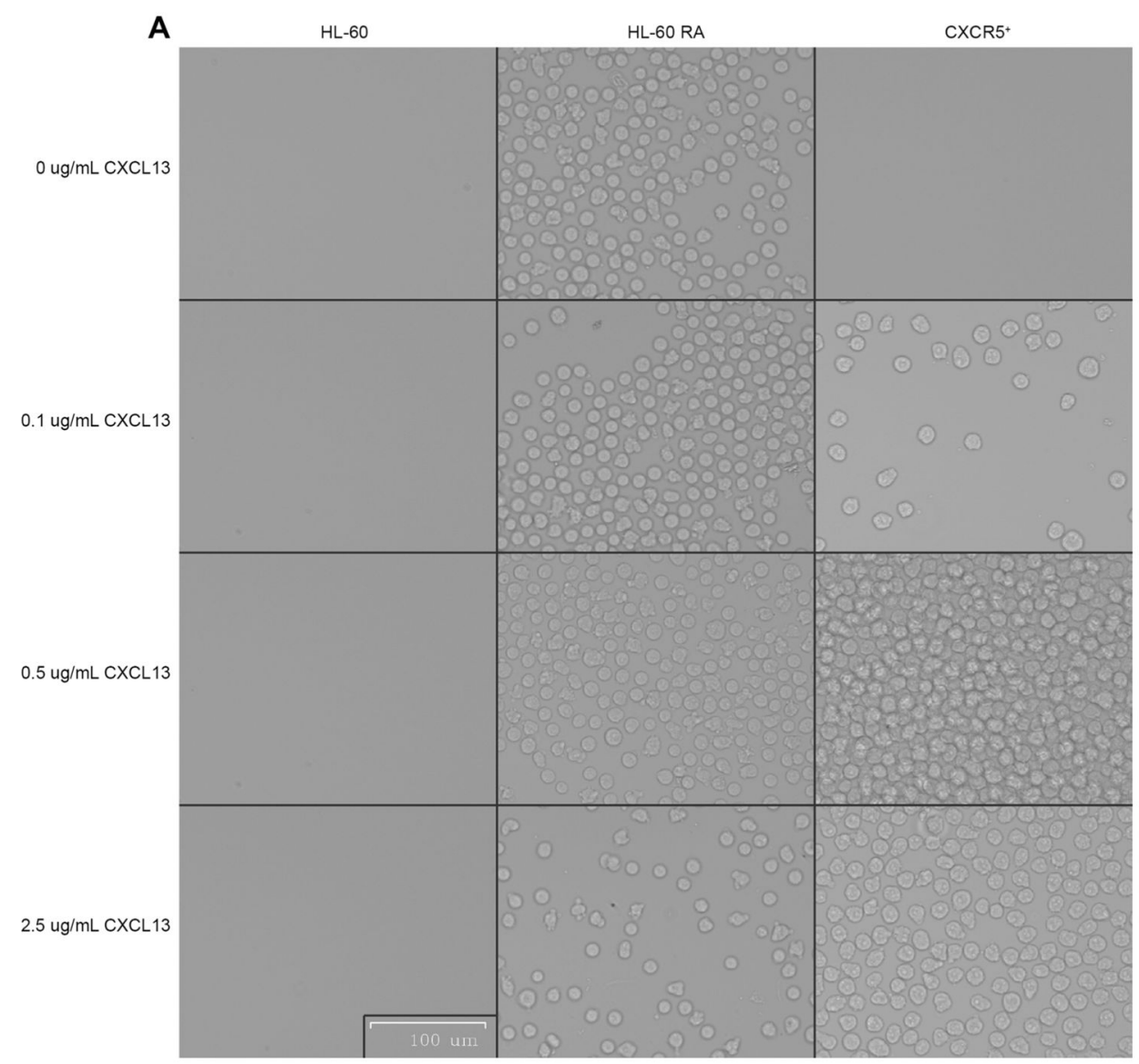

B Percent Migration toward CXCL13

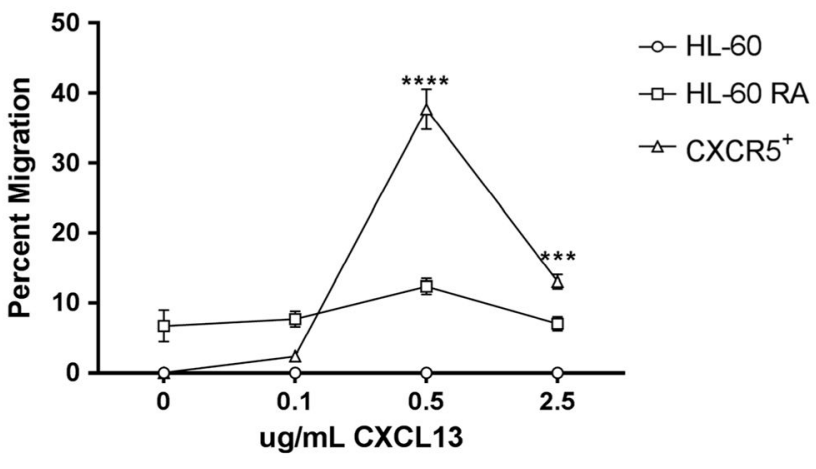

Figure 2.

$\mathrm{CXCR}^{+}$cells exhibit chemotaxis toward CXCL13. (A) HL-60 and CXCR5 ${ }^{+}$cells were cultured with (HL-60 RA) or without (HL-60, CXCR5 ${ }^{+}$) RA for $48 \mathrm{~h}$ and $1.5 \times 10^{5}$ cells were seeded into Transwell membranes over wells containing complete culture media supplemented with CXCL13 $(0.1,0.5$, or $2.5 \mu \mathrm{g} / \mathrm{mL})$ as indicated. After a 6-h incubation period, Transwell inserts were removed, wells were imaged, and cells from the wells were counted. Representative images of microscope fields are shown. $(B)$ Percent migration toward CXCL13 was calculated by dividing counts from the wells by the total number of cells seeded and then multiplying by $100 . n=3$. Error bars indicate SD. ***, $p<0.001$; $* * * *, p<0.0001$. 

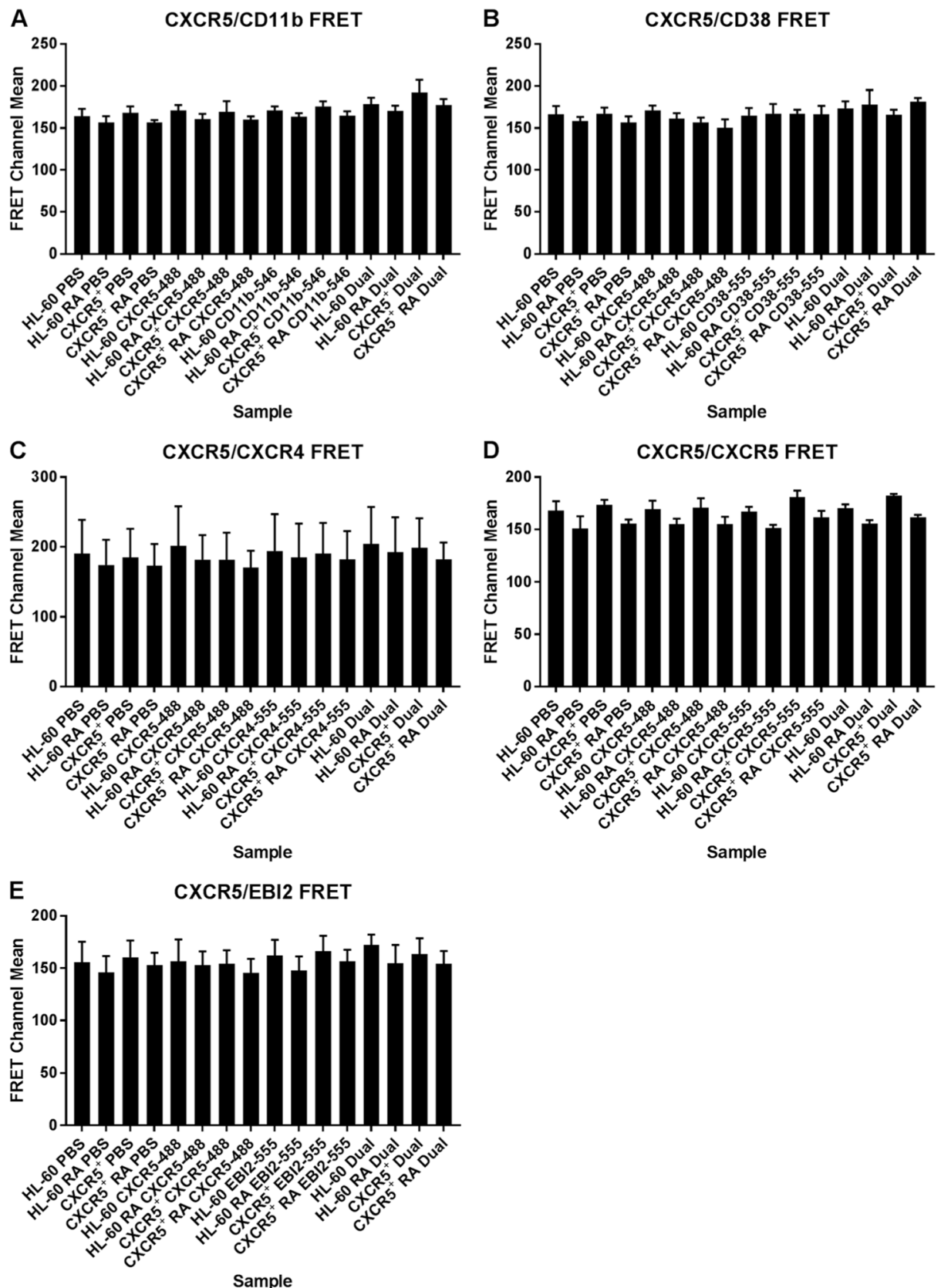

Figure 3.

Flow cytometry FRET between CXCR5 and several membrane proteins. Primary antibodies conjugated to Alexa Fluor 488 (donor) or Alexa Fluor 546/555 (acceptor) were used to label CXCR5 and candidate interaction partners on the cell surface. Mean fluorescence intensity $(n=3)$ in the FRET channel is shown for each pair in HL-60 and CXCR5 ${ }^{+}$cells with or without 24-h RA treatment as indicated. Samples were stained with PBS, single antibodies, or both antibodies (dual). Candidate CXCR5 interaction partners tested were CD11b $(A)$, CD38 (B), CXCR4 (C), CXCR5 (D), and EBI2 (E). 

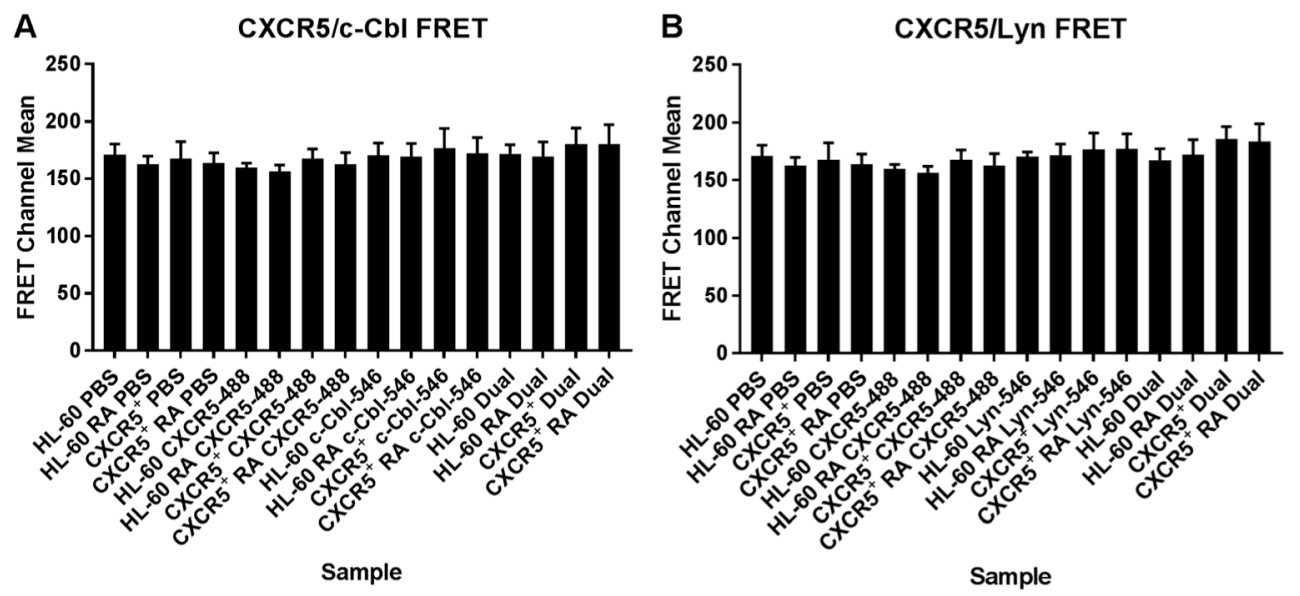

Figure 4.

Flow cytometry FRET between CXCR5 and two cytosolic proteins. Primary antibodies conjugated to Alexa Fluor 488 (donor) or Alexa Fluor 546/555 (acceptor) were used to label CXCR5 and candidate interaction partners on the cell surface. FRET means $(n=3)$ are shown for each pair in HL-60 and $\mathrm{CXCR}^{+}$cells with or without 24-h RA treatment as indicated. Samples were stained with PBS, single antibodies, or both antibodies (dual). Candidate CXCR5 interaction partners tested were c-Cbl $(A)$ and Lyn $(B)$. 


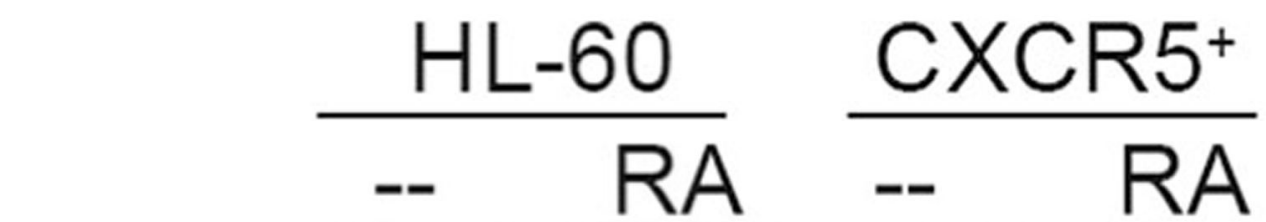

c-Raf
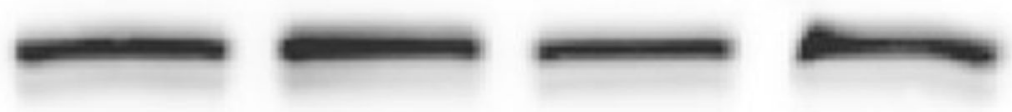

c-Raf pS621

\author{
MEK
}

p-MEK

ERK
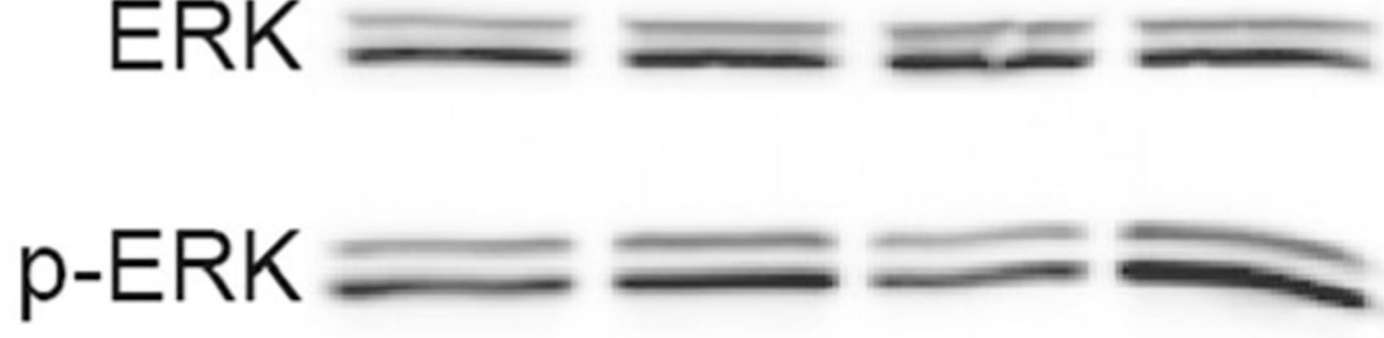

\title{
GAPDH
}

Figure 5.

Levels and phosphorylation status of several MAPK proteins are similar in $\mathrm{CXCR}^{+}$cells and HL-60 cells with and without RA treatment. CXCR5+ and HL-60 cells were treated with $1 \mu \mathrm{M}$ RA for $48 \mathrm{~h}$ as indicated. Lysate was collected and western blots were performed, probing for c-Raf, c-Raf pS621, MEK, pMEK, ERK, and pERK. GAPDH was used as a loading control. $25 \mu \mathrm{g}$ of lysate was loaded per lane; representative images cropped to show the band of interest are shown. $n=3$ biological replicates. 

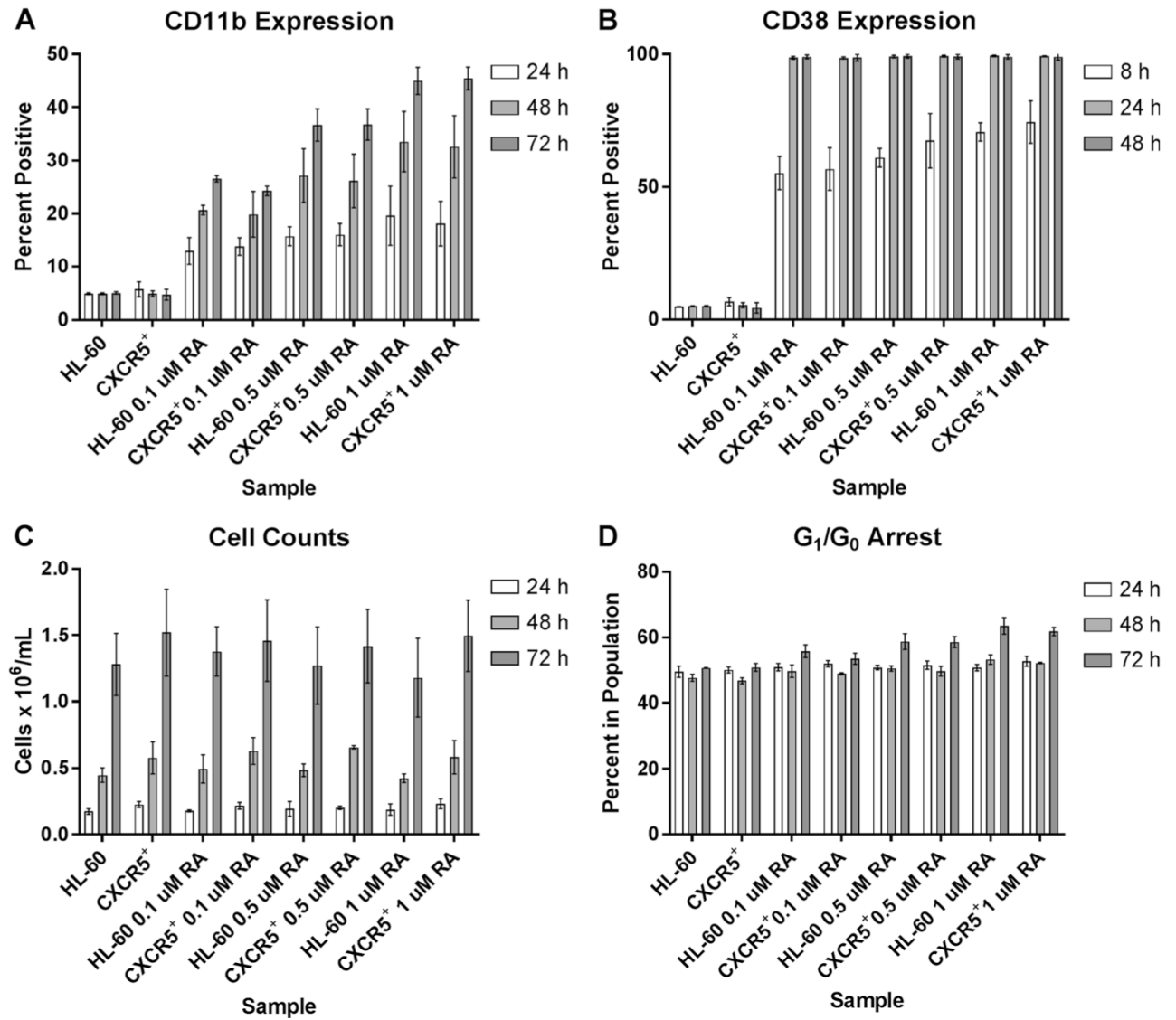

Figure 6.

$\mathrm{CXCR}^{+}$cells differentiate similarly to HL-60 cells upon RA treatment at several concentrations. $(A) \mathrm{HL}-60$ and $\mathrm{CXCR}^{+}$cells were treated with $0.1,0.5$, or $1 \mu \mathrm{M}$ RA over $72 \mathrm{~h}$ as indicated. CD11b expression was assessed by flow cytometry at 24, 48, and $72 \mathrm{~h}$. Gating was set to exclude $95 \%$ of stained control cells (HL-60). (B) CD38 expression was assessed at 8,24, and $48 \mathrm{~h}$. (C) Cell counts were performed using a hemocytometer and $0.2 \%$ trypan blue exclusion staining at 24,48 , and $72 \mathrm{~h}$ after treatment. $(D) \mathrm{G}_{1} / \mathrm{G}_{0}$ cell cycle arrest was measured by flow cytometry using propidium iodide (PI) staining at 24, 48, and 72 h. $n=3$ and error bars indicate SD. 